Original Research Paper

\title{
Exact Classical and Quantum Mechanics of a Generalized Singular Equation of Quadratic Liénard Type
}

\author{
${ }^{1}$ Lucas H. Koudahoun, ${ }^{1}$ Jean Akande, \\ ${ }^{1}$ Damien K.K. Adjaï, ${ }^{2}$ Yélomè J.F. Kpomahou and ${ }^{1}$ Marc D. Monsia \\ ${ }^{1}$ Department of Physics, University of Abomey-Calavi, Abomey-Calavi, 01. BP.526, Cotonou, Benin \\ ${ }^{2}$ Department of Industrial and Technical Sciences, ENSET-Lokossa, University of Abomey, Abomey, Benin
}

\author{
Article history \\ Received: 21-03-2018 \\ Revised: 28-05-2018 \\ Accepted: 19-06-2018 \\ Corresponding Author: \\ Lucas H. Koudahoun \\ Department of Physics, \\ University of Abomey- \\ Calavi, Abomey-Calavi, 01. \\ BP.526, Cotonou, Benin \\ Email: herluckou@gmail.com
}

\begin{abstract}
Authors introduce a generalized singular differential equation of quadratic Liénard type for study of exact classical and quantum mechanical solutions. The equation is shown to exhibit periodic solutions and to include the linear harmonic oscillator equation and the PainlevéGambier XVII equation as special cases. It is also shown that the equation may exhibit discrete eigenstates as quantum behavior under NikiforovUvarov approach after several point transformations.
\end{abstract}

Keywords: Painlevé-Gambier XVII Equation, Liénard Equation, Quantum Mechanics, Point Transformation, Nikiforov-Uvarov Method

\section{Introduction}

A century later, after their elaboration, PainlevéGambier equations continue to be the subject of intense study since their analytical properties and applications are not fully investigated. Ince in his book (Ince, 1956) studied many aspects of properties of these equations by determining first integrals and corresponding analytical solutions. Recently other authors have studied these equations by computing other first integrals in addition to those found by Ince (1956) in order to systematically express exact analytical solutions by the generalized Sundman transformation (Guha et al., 2010). The generalized Sundman transformation is a powerful mathematical tool in the linearization of nonlinear differential equations to facilitate their solving process by the usual analytical methods of integration (Guha et al., 2010; Akande et al. 2017a; Adjaï et al., 2017a). This method of Sundman linearization was recently applied to detect for the first time the existence of a general class of nonlinear differential equations of Liénard type whose solutions are trigonometric (Akande et al. 2017a) but with amplitude dependent frequency. Thus it was allowed to show for the first time that some Painlevé-Gambier equations could admit explicit and exact general trigonometric solutions (Akande et al. 2017a). Recently, this generalized Sundman transformation proposed by Akande et al. (2017a) was used to calculate explicit and exact general periodic solutions of the cubic Duffing equation and of some Painlevé-Gambier equations (Adjaï et al., 2017a). The present work is still interested in the Painlevé-Gambier equations, in particular in Painlevé-Gambier XVII equation. It must be noted, first of all, that the general solution of this equation has been given in the book of Ince (1956). So under these conditions, it remains in analytical investigation of this equation to propose other elegant alternative ways for explicit and exact general solutions, generalized equations or evidence of other properties which are not directly studied by Ince (1956). It is also possible to consider a more general equation which contains the Painlevé-Gambier equation as particular case. Thus, there appears appropriate to investigate the problem to build a generalized equation which admits explicit and exact general solution in terms of trigonometric functions (Adjaï et al., 2017a; Monsia et al., 2016) and contains the linear harmonic oscillator equation and the Painlevé-Gambier XVII equation, as special cases, with applications in physics. In classical mechanics as well as in practical applications, the linear harmonic oscillator equation, as well known, is often used in study of mechanical oscillations (Kpomahou and Monsia, 2015). However, this structural model could not take into consideration, for example, the nonlinear phenomena of energy dissipation in heat and geometrical nonlinearities exhibited by real mechanical systems. As such, nonlinear differential equations with explicit and exact general 
periodic solutions with damping and geometrical nonlinearity properties for which the linear harmonic oscillator equation consists of a limiting case are required in mathematical modeling of mechanical oscillations. So it has been observed that these dissipation phenomena and geometrical nonlinearities may be better captured by oscillators described by quadratic Liénard type equations (Lopèz, 2001; Nonti et al., 2017; Akande et al., 2017b; 2017c; Adjaï et al., 2017b; Rath et al., 2017; Choudoury et al., 2008; Monsia, 2012). These quadratic Liénard type equations may therefore be considered in computing quantum properties of dynamical systems. In addition, such equations exhibit a position dependent mass dynamics, which has been of great importance in improving description of classical and quantum properties of dynamical systems (Akande et al., 2017c; Adjaï et al., 2017b; Rath et al., 2017; Choudoury et al., 2008; Förster et al., 2012). Thus, these harmonic oscillators with a mass varying with distance have been used in many fields of quantum physics as well as engineering applications and are still the subject of intense research activity (Akande et al., 2017c; Adjaï et al., 2017b; Rath et al., 2017; Choudoury et al., 2008; Förster et al., 2012; Schmidt, 2007). However, the Schrödinger equation with a mass varying with distance becomes quickly more complicated to be formulated and solved than the usual Schrödinger equation with a constant mass. The formulation of such a Schrödinger equation is not easy because the usual Hamiltonian operator is no longer Hermitian. One way of overcoming this difficulty is to consider the expression of the Hermitian Hamiltonian of von Roos (1983). But this introduces another complication in the Schrödinger equation known under the ambiguity parameter problem, because there is no rule leading to a rational parametric choice. As soon as this problem is solved, it remains therefore the choice of the appropriate method of solving the Schrödinger eigenvalue equation with a position dependent mass. There is a multitude of techniques for solving such equations in the literature, which proves that there is no standard method that works in all cases of study. Therefore, the question to be solved under these conditions can be announced as follows: Is there a generalized quadratic Liénard type equation admitting the linear harmonic oscillator equation and the PainlevéGambier XVII equation as limiting cases? The identification of this equation may be of great importance given the applications it may offer in physics, especially in classical and quantum mechanics. The nonlinear differential equations with isochronicity are of high interest in physics and engineering applications when dynamical systems with amplitude independent frequency are concerned. Such a question may also enable to show that the nonlinearity behavior of quadratic Liénard type equations affects discrete bound states solutions of corresponding Schrödinger wave equation so that the quantized generalized equation may be used in the control of quantum behavior of dynamical systems, that is to say, as a simulation model of quantum mechanics. It is asserted that this generalized quadratic Liénard type equation exists. Therefore to perform the purpose of this work, the generalized equation under question (Section 2), as well as its explicit and exact general solution in the framework of Riccati variable transformation are established (Section 3). In this way, the exact quantum mechanics of this equation is exhibited under Nikiforov-Uvarov method (1983) after many point transformations (Section 4). Finally a discussion (Section 5 ) and a conclusion for all the work are formulated.

\section{Generalized Equation of Quadratic Liénard Type}

It has recently been shown by Monsia et al. (2016) that some classes of mixed or quadratic Liénard type nonlinear differential equations can be explicitly and exactly solved by application of the Riccati variable transformation. In this study let us consider such a class of equations defined by Monsia et al. (2016):

$\ddot{x}+\left(\frac{a}{h(x)}-\frac{h^{\prime}(x)}{h(x)}\right) \dot{x}^{2}+\frac{4 \omega_{0}^{2}-\lambda^{2}}{4 a} h(x)=0$

in which, $h(x) \neq 0$, is an arbitrary function, $a \neq 0, \omega_{0}$ and $\lambda$ being arbitrary parameters. The dot over a symbol designates a derivative with respect to time and prime means differentiation with respect to position. The problem to be solved under these conditions is to find the appropriate function $h(x)$ to be used to specify (1) as a generalized equation which contains the linear harmonic oscillator equation and the Painlevé-Gambier XVII equation as special cases and having the ability to ensure applications in physics. Imposing $h(x)=m x$, where $m \neq$ 0 , is an arbitrary parameter, the Equation 1 leads to:

$\ddot{x}-\left(\frac{m-a}{m}\right) \frac{\dot{x}^{2}}{x}+\frac{4 \omega_{0}^{2}-\lambda^{2}}{4 a} m x=0$

Making:

$b=\frac{4 \omega_{0}^{2}-\lambda^{2}}{4 a}$

where, $\alpha=\frac{a}{m}$, the Equation 2 may be rewritten:

$\ddot{x}-(1-\alpha) \frac{\dot{x}^{2}}{x}+b x=0$ 
The Equation 2 or 4 is the desired generalized quadratic Liénard type equation. It suffices to note that for the parametric choice $b=0$, that is for $4 \omega_{0}^{2}-\lambda^{2}=0$ and $m \neq 0$, Equation 4 reduces to:

$\ddot{x}-(1-\alpha) \frac{\dot{x}^{2}}{x}=0$

which, for $a=1$, that is $\alpha=\frac{1}{m}$, gives:

$\ddot{x}-\left(\frac{m-1}{m}\right) \frac{\dot{x}^{2}}{x}=0$

known as the Painlevé-Gambier XVII equation (Ince, 1956). As such one may observe the generalized Equation 4 for $a=1$, as the Painlevé-Gambier XVII equation with linear external forcing function $-b x$. For $\alpha$ $=-1$ and $\frac{4 \omega_{0}^{2}-\lambda^{2}}{4}=1$, that is $\lambda=0$ and $\omega_{0}=1$, Equation 2 or 4 becomes the Euler-Lagrange equation corresponding to the alternative Hamiltonian for the harmonic oscillator (Nucci, 2011) while $\alpha=1$, gives the linear harmonic oscillator equation. Such a quadratic Liénard type equation, according to (Nucci, 2011) cannot be correctly quantized by the normalordering and Weyl quantization methods. According to the above the explicit and exact general solution to (4) may be computed and applied to show that it may exhibit periodic oscillations.

\section{Classical Analysis}

This part is devoted to calculate the explicit and exact general solution to (4). It is demonstrated in this way that for an appropriate parametric choice, the general solution to the Painlevé-Gambier XVII equation given by Ince (1956) is recovered by means of the linearizing transformation applied in this research work to find solutions.

\section{Solving of Painlevé-Gambier XVII equation}

To solve explicitly and exactly the Painlevé-Gambier XVII equation, let us consider the Riccati transformation (Monsia et al., 2016):

$\frac{\dot{y}}{y}=a \frac{\dot{x}}{h(x)}$

where, $y \neq 0$. By application of $h(x)=m x$, the Equation 7 reduces by a differentiation with respect to time, taking into consideration (5), to the equation of the free particle motion: $\ddot{y}=0$

with the general solution:

$$
y(t)=k_{1} t+k_{2}
$$

where, $k_{1}$ and $k_{2}$ are arbitrary parameters, so that the general solution to (5) becomes:

$x(t)=\left(k_{1} t+k_{2}\right)^{\frac{1}{\alpha}}$

In this regard, the general solution to the PainlevéGambier XVII equation may, knowing that $a=1$, be written:

$$
x(t)=\left(k_{1} t+k_{2}\right)^{m}
$$

The solution (11) is the same as that given by Ince (1956). It is interesting to note that here, imposing $m=\frac{1}{2}$ and $a=1$, that is $\alpha=2$, Equation 6 reduces to the inverted Painlevé-Gambier XI equation:

$\ddot{x}+\frac{1}{x} \dot{x}^{2}=0$

which admits the general solution:

$x(t)=\left(k_{3} t+k_{4}\right)^{\frac{1}{2}}$

where, $k_{3}$ and $k_{4}$ are constants of integration. Now one may consider the explicit and exact general periodic solution to the generalized quadratic Liénard type equation of interest.

\section{Solving of Generalized Quadratic Liénard Type Equation}

By applying the Riccati transformation (7) with $h(x)$ $=m x$, the explicit and exact general periodic solution of (4) may be expressed as:

$x(t)=A^{\frac{1}{\alpha}} \sin ^{\frac{1}{\alpha}}\left(\sqrt{\frac{4 \omega_{0}^{2}-\lambda^{2}}{4}} t+K\right)$

where, $A$ and $K$ are arbitrary parameters and $\frac{4 \omega_{0}^{2}-\lambda^{2}}{4}>0$. It is interesting to note that such a solution is periodic with amplitude independent frequency, so that for $\frac{4 \omega_{0}^{2}-\lambda^{2}}{4}>0$, the generalized Equation 4 may exhibit isochronicity property. For $\alpha=1$, the Equation 4 
reduces to that of the harmonic oscillator for $\lambda=0$, such that the explicit and exact general periodic solution (14) becomes a trigonometric solution with fixed frequency, that is with amplitude independent frequency. An interesting case is also when the exponent $\frac{1}{\alpha}=\frac{m}{a}$, in the expression (14) is a positive integer greater than or equal to two, allowing to write the solution (14) as a linear combination of terms having the form $\sin (q t+\varphi)$ or $\cos$ $(q t+\varphi)$ where $q$ denotes a positive integer and $\varphi$ is an arbitrary parameter. Under these conditions, the solution (14) assumes for $\alpha=\frac{1}{2}$, a very interesting expression:

$$
x(t)=\frac{A^{2}}{2}-\frac{A^{2}}{2} \cos \left(\sqrt{4 \omega_{0}^{2}-\lambda^{2}} t+C\right)
$$

where, $C=2 K$. The solution (15) is a trigonometric solution like that of the harmonic oscillator but with a shift factor $\frac{A^{2}}{2}$. That being so the quantum mechanics of the generalized quadratic Liénard type Equation 4 may be carried out.

\section{Quantum Mechanical Analysis}

As mentioned in the above, it is interesting to show that the generalized Equation 4 may be quantized in a perspective where it may be used in physical and practical applications to compute the discrete bound states of dynamical systems.

\section{Schrödinger Eigenvalue Problem}

The objective of this part is to establish the Schrödinger wave equation in question. This will be carried out under the formalism of Schrödinger equation with position-dependent mass. Given (4), the mass distribution function $M(x)$ may be calculated as (Mustafa, 2015):

$$
M(x)=e^{-2 \int(1-\alpha) d x}
$$

that is:

$$
M(x)=m_{0} x^{2(1-\alpha)}
$$

so that the potential function using the formula:

$$
V(x)=b \int x M(x) d x
$$

takes the form:

$$
V(x)=\frac{1}{2 \alpha} m_{0} b x^{2 \alpha}
$$

In this way the position dependent mass Schrödinger eigenvalue problem for the potential (17) with the mass (16) to be solved may clearly be stated.

\section{Problem Description}

Let us consider a particle described by (4) of mass $M(x)$, moving in the potential $V(x)$. The problem is to find in this context, the Schrödinger wave function $\psi(x)$ and energy eigenvalue $E$ under the conditions that $\psi(x)$ is bounded and of square integrable on the interval $\left[0, A^{\frac{1}{\alpha}}\right]$. According to (Akande et al., 2017b; 2017c; Adjaï et al., $2017 b)$ the Schrödinger eigenvalue problem which has been described previously may be written in the form:

$\psi^{\prime \prime}(x)-\frac{M^{\prime}(x)}{M(x)} \psi^{\prime}(x)+2 M(x)[E-V(x)] \psi(x)=0$

where, prime designates the derivative with respect to position. Substituting the Equations 16 and 17, when $\beta=\frac{b}{\alpha}$ where, $\alpha \neq 0$, into (18) yields the desired Schrödinger equation with position dependent mass:

$$
\begin{aligned}
& \psi^{\prime \prime}(x)-\frac{(2 \alpha-2)}{x} \psi^{\prime}(x)+ \\
& {\left[2 E x^{2(\alpha-1)}-\beta x^{2(2 \alpha-1)}\right] \psi(x)=0}
\end{aligned}
$$

where, $m_{0}=1$ and $b=\frac{4 \omega_{0}^{2}-\lambda^{2}}{4 \alpha}$, over the interval $0 \leq x \leq A^{\frac{1}{\alpha}}$ with $\psi(0)=\psi\left(A^{\frac{1}{\alpha}}\right)=0$. It is interesting to mention that, using the Liouville transformation (Fédoriouk, 1987):

$\psi(x)=\varphi(x) x^{\alpha-1}$

the equation (19) may be reduced to the normal form:

$$
\begin{aligned}
& \varphi^{\prime \prime}(x) \\
& +\left[2 E x^{2(\alpha-1)}-\beta x^{2(2 \alpha-1)}-\alpha(\alpha-1) \frac{1}{x^{2}}\right] \varphi(x)=0
\end{aligned}
$$

Now to determine the eigensolutions, it is needed to write (21) in an appropriate hypergeometric type equation.

\section{Mapping of (21) into Hypergeometric Type Equation}

The objective in this subsection is to map the Equation 21 into an appropriate form in order to secure the desired eigensolutions. To this end, it is convenient 
to reduce (21) to an appropriate normal form which may enable to deduce an exactly solvable hypergeometric type equation. Putting:

$$
Q(x)=2 E x^{2(\alpha-1)}-\beta x^{2(2 \alpha-1)}-\alpha(\alpha-1) \frac{1}{x^{2}}
$$

Equation 21 may be written as:

$$
\varphi^{\prime \prime}(x)+Q(x) \varphi(x)=0
$$

The application of the point canonical transformation (Fédoriouk, 1987):

$$
x=h(z), \varphi(x)=\left[h^{\prime}(z)\right]^{\frac{1}{2}} u(z)
$$

reduces (23) to:

$$
u^{\prime \prime}(z)+\left[\begin{array}{l}
\frac{h^{\prime \prime \prime}(z)}{2 h^{\prime}(z)}-\frac{3}{4}\left(\frac{h^{\prime \prime}(z)}{h^{\prime}(z)}\right)^{2} \\
+Q(h(z))\left[h^{\prime}(z)\right]^{2}
\end{array}\right] u(z)=0
$$

Now let us consider:

$$
h(z)=z^{\ell}
$$

where, $\ell$ is an arbitrary parameter. The substitution of (26) into (25) yields the normal form:

$$
\begin{aligned}
& u^{\prime \prime}(z) \\
& +\left[\begin{array}{l}
2 E \ell^{2} z^{(2 \ell \alpha-2)}-\beta \ell^{2} z^{(4 \ell \alpha-2)} \\
-\frac{\alpha(\alpha-1) \ell^{2}-\frac{1}{2}(\ell-1)(\ell-2)+\frac{3}{4}(\ell-1)^{2}}{z^{2}}
\end{array}\right]
\end{aligned}
$$$$
\times u(z)=0
$$

As $\ell$ is an arbitrary parameter, it is always possible to set the product $\ell \alpha$ to an arbitrary value. Setting $\ell \alpha=1$, that is $\ell=\frac{1}{\alpha}$, Equation 27 therefore becomes:

$$
u^{\prime \prime}(z)+\left[\begin{array}{l}
\frac{2 E}{\alpha^{2}}-\frac{\beta}{\alpha^{2}} z^{2} \\
-\frac{1}{4}\left(\frac{1}{\alpha}-3\right)\left(\frac{1}{\alpha}-1\right) \frac{1}{z^{2}}
\end{array}\right] u(z)=0
$$

which may be mapped into a solvable hypergeometric type equation. For the purpose to apply the NikiforovUvarov method to compute the exact discrete bound state solutions, Equation 28 must be transformed into an appropriate form which supports such an approach. Thus under the variable transformation:

$s=z^{2}$

Equation 28 takes the desired form:

$$
\begin{aligned}
& u^{\prime \prime}(s)+\frac{1}{2 s} u^{\prime}(s) \\
& +\frac{1}{s^{2}}\left[-\frac{\beta}{4 \alpha^{2}}+\frac{E}{2 \alpha^{2}} s-\frac{1}{16}\left(\frac{1}{\alpha^{2}}-\frac{4}{\alpha}+3\right)\right] u(s)=0
\end{aligned}
$$

which may be solved by applying the Nikiforov-Uvarov (1983) to ensure the discrete eigenstates.

\section{Application of the Nikiforov-Uvarov method}

The Nikiforov-Uvarov method has been widely used by several authors (Akande et al., 2017c; Adjaï et al., 2017b; Abdalla and Eleuch, 2016; Buyukilic et al., 1997) to solve exactly the Schrödinger wave equation with position-dependent mass. This method is very interesting due to its simplicity and elegance in solving process steps. The Nikiforov-Uvarov method is usually applied to solve the general second order linear equation (Nikiforov and Uvarov, 1983):

$u^{\prime \prime}(s)+\frac{\tilde{\tau}(s)}{\sigma(s)} u^{\prime}(s)+\frac{\tilde{\sigma}(s)}{\sigma(s)^{2}} u(s)=0$

where, $\tilde{\tau}(s)$ is a polynomial at most of first degree while $\sigma(s)$ and $\tilde{\sigma}(s)$ are polynomials at most of second degree. In this regard the wave function $u(s)$ is expressed as:

$u(s)=\phi(s) y_{n}(s)$

where the function $y_{n}(s)$ satisfies the hypergeometric type linear differential equation:

$\sigma(s) y_{n}^{\prime \prime}(s)+\tau(s) y_{n}^{\prime}(s)+\lambda y_{n}(s)=0$

and:

$\frac{\phi^{\prime}(s)}{\phi(s)}=\frac{\pi(s)}{\sigma(s)}$

under the condition that $\tau(s)$ should be a polynomial at most of first degree, $\lambda$ is a constant and $\pi(s)$ satisfies:

$$
\begin{aligned}
& \pi(s)=\frac{\sigma^{\prime}(s)-\tilde{\tau}(s)}{2} \\
& \pm \sqrt{\left(\frac{\sigma^{\prime}(s)-\tilde{\tau}(s)}{2}\right)^{2}-\tilde{\sigma}(s)+k \sigma(s)}
\end{aligned}
$$


The polynomial $\pi(s)$ is required to be at most of first degree with the conditions that:

$\tau(s)=\tilde{\tau}(s)+2 \pi(s)$

$k=\lambda-\pi^{\prime}(s)$

and:

$\lambda=\lambda_{n}=-n \tau^{\prime}(s)-\frac{n(n-1)}{2} \sigma^{\prime \prime}(s), n=0,1,2,3, \ldots$

As such the hypergeometric-type function $y_{n}(s)$ is considered as a polynomial of degree $n$ with an expression given by the Rodrigues formula:

$y_{n}(s)=\frac{A_{n}}{\rho(s)} \frac{d^{n}}{d s^{n}}\left[\sigma(s)^{n} \rho(s)\right]$

in the sense that the weight function $\rho(s)$ satisfies:

$\frac{d}{d s}[\sigma(s) \rho(s)]=\tau(s) \rho(s)$

and $A_{n}$ denotes the normalization constant. In the context where $\sigma(s)$ $=$ $S$, $\tilde{\tau}(s)=\frac{1}{2}, \tilde{\sigma}(s)=-\frac{\beta}{4 \alpha^{2}} s^{2}+\frac{E}{2 \alpha^{2}} s-\frac{1}{16}\left(\frac{1}{\alpha^{2}}-\frac{4}{\alpha}+3\right), \quad$ the polynomial $\pi(s)$ may take the expression:

$$
\begin{aligned}
& \pi(s)=\frac{1}{4} \\
& \pm \sqrt{\frac{\beta}{4 \alpha^{2}} s^{2}+\left(k-\frac{E}{2 \alpha^{2}}\right) s+\frac{1}{16}\left(\frac{1}{\alpha^{2}}-\frac{4}{\alpha}+4\right)}
\end{aligned}
$$

which becomes under the requirement that the derivative of $\tau(s)$ must be negative:

$$
\pi(s)=\pi_{+}(s)=\frac{1}{4}-\frac{\sqrt{\beta}}{2 \alpha^{2}} s-\frac{1}{4}\left(\frac{1}{\alpha}-2\right)
$$

where:

$$
k=k_{+}=\frac{E}{2 \alpha^{2}}+\frac{\sqrt{\beta}}{4 \alpha}\left(\frac{1}{\alpha}-2\right)
$$

or:

$$
\pi(s)=\pi_{-}(s)=\frac{1}{4}-\frac{\sqrt{\beta}}{2 \alpha} s+\frac{1}{4}\left(\frac{1}{\alpha}-2\right)
$$

for:

$$
k=k_{-}=\frac{E}{2 \alpha^{2}}-\frac{\sqrt{\beta}}{4 \alpha}\left(\frac{1}{\alpha}-2\right)
$$

In such a situation:

$\tau(s)=\tilde{\tau}(s)+2 \pi(s)$

reduces to:

$$
\tau(s)=\tau_{+}(s)=1-\frac{1}{2}\left(\frac{1}{\alpha}-2\right)-\frac{\sqrt{\beta}}{\alpha} s
$$

for $\pi_{+}(s)$, or:

$$
\tau(s)=\tau_{-}(s)=1+\frac{1}{2}\left(\frac{1}{\alpha}-2\right)-\frac{\sqrt{\beta}}{\alpha} s
$$

for $\pi_{-}(s)$. Therefore two cases must be considered in computing eigensolutions.

\section{Discrete Bound State Solutions for $k_{+}$}

This paragraph deals with the determination of exact discrete bound state energy spectrum and wave functions of the dynamical system under consideration for $k_{+}$.

\section{Discrete Bound State Energy Eigenvalues}

The substitution of $\tau_{+}(s)$ and $\sigma(s)$ into (38) leads to:

$$
\lambda_{n}=n \frac{\sqrt{\beta}}{\alpha}
$$

On the other hand, the use of (43) by taking into consideration (37) yields:

$\lambda_{n}=\frac{E}{2 \alpha^{2}}+\frac{\sqrt{\beta}}{2 \alpha}\left(\frac{1}{2 \alpha}-2\right)$

Therefore the comparison of (49) with (50) provides the desired exact discrete energy eigenvalues:

$E_{n}=\frac{\sqrt{\beta}}{2}[4 \alpha(n+1)-1], n=0,1,2, \ldots$

Since $\quad \alpha=\frac{a}{m} \quad$ and $\beta=\frac{m^{2}\left(4 \omega_{0}^{2}-\lambda^{2}\right)}{4 a^{2}}$, the energy eigenvalues $E_{n}$ become:

$$
E_{n}=\left[n+1-\frac{m}{4 a}\right] \sqrt{4 \omega_{0}^{2}-\lambda^{2}}, n=0,1,2, \ldots
$$

Equation 52 is very interesting since it shows that the energy spectrum depends not only on natural frequency 
$\omega_{0}$ as in the case of the quantum harmonic oscillator, but also on nonlinearity parameter $\alpha=\frac{a}{m}$ of the classical model. In this way the discrete bound state wave functions may be exactly calculated.

\section{Discrete Bound State Wave Functions}

According to the above, the function $\phi(s)$ defined by (34) may be computed under the form:

$$
\phi(s)=s^{\left(\frac{3}{4}-\frac{1}{4 \alpha}\right)} e^{-\frac{\sqrt{\beta}}{2 \alpha} s}
$$

so that the hypergeometric type function $u_{n}(s)$ may take the expression:

$$
u_{n}(s)=A_{n} s^{\frac{1}{4}\left(\frac{1}{\alpha}-1\right)} e^{\frac{\sqrt{\beta}}{2 \alpha}} s \frac{d^{n}}{d s^{n}}\left(s^{n+1-\frac{1}{2 \alpha}} e^{-\frac{\sqrt{\beta}}{\alpha} s}\right)
$$

Using the definition of the associated Laguerre polynomials (Arfken and Weber, 2005; Szegö, 1939):

$$
L_{n}^{r}(\tau)=\frac{1}{n !} e^{\tau} \tau^{-r} \frac{d^{n}}{d \tau^{n}}\left(\tau^{n+r} e^{-\tau}\right)
$$

Equation 54 may be written as:

$$
u_{n}(s)=B_{n} s^{\frac{1}{4}\left(3-\frac{1}{\alpha}\right)} e^{-\frac{\sqrt{\beta}}{2 \alpha} s} L_{n}^{1-\frac{1}{2 \alpha}}\left(\frac{\sqrt{\beta}}{\alpha} s\right)
$$

where, $B_{n}$ denotes a normalization constant. Knowing $s=$ $z^{2},(56)$ becomes:

$$
u_{n}(z)=B_{n} z^{\frac{1}{2}\left(3-\frac{1}{\alpha}\right)} e^{-\frac{\sqrt{\beta}}{2 \alpha} z^{2}} L_{n}^{1-\frac{1}{2 \alpha}}\left(\frac{\sqrt{\beta}}{\alpha} z^{2}\right)
$$

In this context the wave function $\varphi(x)$, taking into consideration (24), may read:

$$
\varphi(x)=C_{n} x^{\alpha} e^{-\frac{\sqrt{\beta}}{2 \alpha} x^{2 \alpha}} L_{n}^{1-\frac{1}{2 \alpha}}\left(\frac{\sqrt{\beta}}{\alpha} x^{2 \alpha}\right)
$$

where, $z=x^{\alpha}$, so that the initial wave function $\psi(x)=$ $x^{\alpha-1} \varphi(x)$, takes definitively the expression:

$$
\psi(x)=C_{n} x^{2 \alpha-1} e^{-\frac{\sqrt{\beta}}{2 \alpha} x^{2 \alpha}} L_{n}^{1-\frac{1}{2 \alpha}}\left(\frac{\sqrt{\beta}}{\alpha} x^{2 \alpha}\right)
$$

where, it is required that:

$$
L_{n}^{1-\frac{1}{2 \alpha}}\left(\frac{\sqrt{\beta}}{\alpha} A^{2}\right)=0
$$

As $\psi_{n}(0)=\psi_{n}\left(A^{\frac{1}{\alpha}}\right)=0, \alpha>\frac{1}{2}$ and $C_{n}$ is the new normalization constant determined by:

$$
\int_{0}^{4^{\frac{1}{\alpha}}}\left|\psi_{n}(x)\right|^{2} d x=1
$$

Equation 60 shows that the nonlinearity parameter $\alpha=\frac{a}{m}$, that is the energy eigenvalues $E_{n}$ are related to the appropriate associated Laguerre polynomials zeros, so that $\alpha$ depends on eigenstates and may be noted $\alpha=\alpha_{n}, n=1,2, \ldots$. In such a condition, $n \geq 1$, the first eigenvalue may take for $\alpha_{1}=\frac{1+\left[1+16 A^{2} \sqrt{4 \omega_{0}^{2}-\lambda^{2}}\right]^{\frac{1}{2}}}{8}$, the expression:

$$
E_{1}=\frac{2 \sqrt{4 \omega_{0}^{2}-\lambda^{2}}\left[1+16 A^{2} \sqrt{4 \omega_{0}^{2}-\lambda^{2}}\right]^{\frac{1}{2}}}{1+\left[1+16 A^{2} \sqrt{4 \omega_{0}^{2}-\lambda^{2}}\right]^{\frac{1}{2}}}
$$

So with that the discrete bound state solutions for the case $k=k_{-}$may be performed.

\section{Discrete bound state solutions for $k=k_{-}$}

Using the same approach as previously, the exact discrete bound state solutions in the case $k=k_{-}$, may be easily computed.

\section{Discrete Bound State Energy Eigenvalues}

To compute the discrete bound state energy spectrum it suffices to calculate again $\lambda_{n}$ by introducing $\tau_{-}(s)$ and $\sigma(s)$ into (38), which gives:

$$
\lambda_{n}=n \frac{\sqrt{\beta}}{\alpha}
$$

and to compare the result with $\lambda_{n}$ given by (45), that is:

$$
\lambda_{n}=\frac{E}{2 \alpha^{2}}-\frac{\sqrt{\beta}}{4 \alpha^{2}}
$$

Therefore one may express the energy eigenvalues as:

$$
E_{n}=2 \alpha \sqrt{\beta}\left(n+\frac{1}{4 \alpha}\right)
$$

that is:

$$
E_{n}=\left(n+\frac{m}{4 a}\right) \sqrt{4 \omega_{0}^{2}-\lambda^{2}}, n=0,1,2, \ldots
$$


Here again the energy spectrum depends on $\frac{m}{a}$, which characterises the nonlinearity property of the investigated dynamical system. After computing the eigenvalues one may determine now the corresponding discrete wave functions.

\section{Discrete Bound State Wave Functions}

Applying the same procedure as in the case $k=k_{+}$, the eigenfunctions $u_{n}(s)$ for $k=k_{-}$, if $D_{n}$ is a normalization constant, may be expressed in the form:

$$
u_{n}(s)=D_{n} s^{\frac{1}{4}\left(3-\frac{1}{\alpha}\right)} e^{\frac{\sqrt{\beta}}{2 \alpha}} \frac{d^{n}}{d s^{n}}\left(s^{n+\frac{1}{2 \alpha}-1} e^{-\frac{\sqrt{\beta}}{\alpha} s}\right)
$$

which may lead, using the identity $s=z^{2}$, to:

$$
u_{n}(z)=B_{n} z^{\frac{1}{2}\left(\frac{1}{\alpha}-1\right)} e^{-\frac{\sqrt{\beta}}{2 \alpha} z^{2}} L_{n}^{\frac{1}{2 \alpha}-1}\left(\frac{\sqrt{\beta}}{\alpha} z^{2}\right)
$$

so that the wave functions $\varphi(x)$ may take the expression:

$$
\varphi_{n}(x)=N_{n} x^{1-\alpha} e^{-\frac{\sqrt{\beta}}{2 \alpha} x^{2 \alpha}} L_{n}^{\frac{1}{2 \alpha}-1}\left(\frac{\sqrt{\beta}}{\alpha} x^{2 \alpha}\right)
$$

where, $N_{n}$ designates the new normalization constant. In such a situation, the initial wave functions $\psi_{n}(x)$ may be in definitive expressed in the form:

$$
\psi_{n}(x)=N_{n} e^{-\frac{\sqrt{\beta}}{2 \alpha} x^{2 \alpha}} L_{n}^{\frac{1}{2 \alpha}-1}\left(\frac{\sqrt{\beta}}{\alpha} x^{2 \alpha}\right)
$$

Since $\alpha>0, \quad L_{n}^{\frac{1}{2 \alpha}-1}(0) \neq 0$, such that $(70)$ cannot be eigensolutions to the boundary value problem under consideration. However, for $\alpha \rightarrow \infty$, one may have $L_{n}^{-1}(0)=0$, according to (Szegö, 1939), where $n \geq 1$, so that (70) may consist of eigenfunctions to the eigenvalue problem investigated. In this case, $L_{n}^{\frac{1}{2 \alpha}-1}\left(\frac{\sqrt{\beta}}{\alpha} x^{2 \alpha}\right)$ must be replaced by (Szegö, 1939):

$$
\left(-\frac{\sqrt{\beta}}{\alpha} x^{2 \alpha}\right) \frac{(n-1) !}{n !} L_{n-1}^{1}\left(\frac{\sqrt{\beta}}{\alpha} x^{2 \alpha}\right)
$$

\section{Discussion}

Many physical processes are studied on the basis of nonlinear differential equations. Conversely, the construction of nonlinear differential equations may be useful in the conception of new dynamical systems for engineering applications. As such a quadratic Liénard type nonlinear dynamical system which contains the Painlevé-Gambier XVII equation, the harmonic oscillator equation and its alternative form as special cases has been constructed. In so doing, it has been possible to show that an usual change of dependent variable, that is, a simple Riccati transformation is enough sufficient to secure the general solution to the Painlevé-Gambier XVII equation which agrees with that found by Ince (1956). In this perspective it is shown that the performed generalized equation may be of interest for some physical applications. Indeed this generalized equation exhibits periodic oscillations with isochronicity characteristic consisting of a $\frac{m}{a} t h$ power of a trigonometric function. As expected, this general behavior reduces to that of the linear harmonic oscillator by putting $\frac{m}{a}=1$ and $\lambda=0$. This fact enables then to investigate the quantum mechanics of the developed generalized equation. Such an investigation is reasonable since it is well known that only few nonlinear differential equations are quantum mechanics solvable problems. In such a way the problem quickly becomes more complicated when quadratic damping effects are considered. So the quantum mechanics of a quadratic Liénard type differential equation is not a simple task. However, fortunately under the formalism of Schrödinger equation with position dependent mass, exact discrete bound state solutions in terms of well known associated Laguerre polynomials are obtained for the quantization of the generalized second order singular equation of quadratic Liénard type investigated on the basis of the Nikiforov-Uvarov approach for solving hypergeometric type differential equations after several point transformations. Such an approach enables to compute the discrete bound state solutions in terms of the nonlinearity parameter $\frac{1}{\alpha}$. In this regard, the Nikiforov-Uvarov method appeared to be an advanced and efficient mathematical technique in solving the Schrödinger wave equation in terms of special orthogonal functions as noticed in several papers (Akande et al., 2017c; Adjaï et al., 2017b; Buyukilic et al., 1997). However the class of singular equations defined by $\alpha<\frac{1}{2}$ cannot be quantized by the present theory so that this opens up new research perspectives as to their quantization. This fact agrees well with $\alpha=-1$, which gives the well-known alternative form for the linear harmonic oscillator equation, for which the application of normal-ordering and Weyl quantization techniques leads to incorrect physical results (Nucci, 2011). In this perspective it may be possible by applying other techniques like matrix methods and in particular the 
Foldy-Wouthuysen transformation method (Khan, 2006; 2008) to solve the considered differential equation for comparison purpose. So according to the above, a conclusion may be carried out for this work.

\section{Conclusion}

This work introduces a generalized second order singular differential equation of quadratic Liénard type which contains the Painlevé-Gambier XVII equation and the linear harmonic oscillator with well-known solutions as special cases. The utility of the equation has been highlighted through its possible applications in classical and quantum mechanics. In so doing it has been possible to show that the introduced generalized equation may exhibit exact periodic oscillations with amplitude independent frequency and discrete bound state solutions through quantization. It is found that eigensolutions depend on amplitude of oscillations of the dynamical system so that such a property may be used in quantum applications.

\section{Author's Contributions}

All authors contributed to the development and formulation of this work. All authors read and approved the final manuscript for publication.

\section{Ethics}

The authors declare that there exists no competing interests.

\section{References}

Abdalla, M.S. and H. Eleuch, 2016. Exact solutions of the position-dependent-effective mass Schrödinger equation. AIP Adv., 6: 055011-1- 055011-7. DOI: $10.1063 / 1.4949567$

Adjaï, D.K.K., L.H. Koudahoun, J. Akande, Y.J.F. Kpomahou and M.D. Monsia, 2017a. On analysis of a class of quadratic Liénard type equations exhibiting exact periodic solutions. viXra: $1705.0262 \mathrm{v} 2$.

Adjaï, D.K.K., J. Akande, L.H. Koudahoun, Y.J.F. Kpomahou and M.D. Monsia, 2017b. Quantum mechanics of singular inverse square potential under usual boundary conditions. viXra: 1703.0101v1.

Akande, J., D.K.K. Adjaï, L.H. Koudahoun, Y.J.F. Kpomahou and M.D. Monsia, 2017a. Theory of exact trigonometric periodic solutions to quadratic Liénard type equations. viXra: 1704.0199v3.

Akande, J., D.K.K. Adjaï, L.H. Koudahoun, Y.J.F. Kpomahou and M.D. Monsia, 2017b. Prolate spheroidal wave function as exact solution of the Schrödinger equation. viXra: 1701.0166v1.
Akande, J., D.K.K. Adjaï, L.H. Koudahoun, B. Rath and P. Mallick et al., 2017c. Exact quantum mechanics of quadratic Liénard type oscillator equations with bound states energy spectrum. viXra: $1702.0242 \mathrm{v} 1$.

Arfken, G.B. and H.J. Weber, 2005. Mathematical Methods for Physicists International Student Edition. 1st Edn., Elsevier, Amsterdam, ISBN-10: 0080470696, pp: 1200.

Buyukilic, F., H. Egrifes and D. Demirhan, 1997. Solution of the Schrödinger equation for two differential molecular potentials by the NikiforovUvarov method. Theor. Chem. Acc., 98: 192-196.

Choudoury, A.G., P. Guha and B. Khanra, 2008. Solutions of some second order ODEs by the extended Prelle-Singer method and symmetries. J. Nonlinear Math. Phys., 15: 365-382. DOI: $10.2991 /$ jnmp.2008.15.4.2

Fédoriouk, M., 1987. Méthodes asymptotiques pour les équations différentielles ordinaires linéaires.

Förster, J., A. Saenz and U. Wolff, 2012. Matrix algorithm for solving Schrödinger equations with position-dependent mass or complex optical potentials. Phys. Rev. E, 86: 016701-1-016701-9. DOI: 10.1103/PhysRevE.86.016701

Guha, P., B. Khanra and A.G. Choudoury, 2010. On generalized Sundman transformation method, first integrals, symmetries and solutions of equations of Painlevé-Gambier type. Nonlinear Anal., 72: 32473257. DOI: 10.1016/j.na.2009.12.004

Ince, E.L. 1956. Ordinary Differential Equations. 1st Edn., Dover, New York, ISBN-10: 0486603490, pp: 558.

Khan, S.A., 2006. The Foldy-Wouthuysen transformation technique in optics. Int. J. Light Electron Opt., 117: 481-488.

DOI: $10.1016 /$ j.ijleo.2005.11.010

Khan, S.A., 2008. The Foldy-Wouthuysen transformation technique in Optics. In: Advances in Imaging and Electron Physics, Hawkes, P.W. (Ed.), ISSN-10: 1076-5670, pp: 49-78.

Kpomahou, Y. J. F. and M. D. Monsia 2015. Hardening nonlinearity effects on forced vibration of viscoelastic dynamical systems to external step perturbation field. Int. J. Adv. Applied Math. Mech., 3: 107-124.

Lopèz, G., 2001. Restricted constant of motion for the one-dimensional harmonic oscillator with quadratic dissipation and some consequences in statistic and quantum mechanics. Int. J. Theor. Phys., 40: 17991807. DOI: 10.1023/A:1011972700121

Monsia, M. D., J. Akande, D. K. K. Adjaï, L.H. Koudahoun and Y. J. F. Kpomahou 2016. A theory of exactly integrable quadratic Liénard type equations. Int. J. Adv. Applied Math. Mech., 4: 21-25.

Monsia, M.D., 2012. A mathematical model for predicting the relexation of creep strains in materials. Phys. Rev. Res. Int., 2: 107-124. 
Mustafa, O., 2015. Position-dependent Lagrangians: nonlocal transformations, Euler-Lagrange invariance and exact solvability. arXiv: 1411.4405v3.

Nikiforov, A. and V. Ouvarov, 1983. Fonctions Spéciales de la physique mathématique.

Nonti, M., F.J.Y. Kpomahou, J. Akande, D.K.K. Adjaï and L.H. Koudahoun et al., 2017. Exact Gibbs statistical mechanics of a system of quadratic Liénard type oscillators. viXra: $1706.0137 \mathrm{v} 2$.

Nucci, M.C. 2011. Quantization of classical mechanics: Shall We Lie. Theoretical Math. Phys., 168: 994-1001. DOI: $10.1007 / \mathrm{s} 11232-011-0081-3$
Rath, B., P. Mallick, J. Akande, D.K.K. Adjaï and L.H. Koudahoun et al., 2017. A general type of Liénard second order differential equation: Classical and quantum mechanical study. Proc. Indian Nat. Sci. Acad., 83: 935-940.

Roos, O.V., 1983. Position-dependent effective masses in semiconductor theory. Phys. Rev. B, 27: 7547-7547. DOI: 10.1103/PhysRevB.27.7547

Schmidt, A. G. M. 2007. Time evolution for harmonic oscillators with position dependent mass. Phys. Scr., 75: 480-483. DOI: 10.1088/0031-8949/75/4/019

Szegö, G., 1939. Orthogonal Polynomials. 3rd Edn., American Mathematical Society, Providence, pp: 401. 